\section{Out-of-hospital noninvasive ventilation: epidemiology, technology and equipment}

\author{
John Scott Baird, Thyyar M. \\ Ravindranath \\ Division of Pediatric Critical Care, \\ Department of Pediatrics, Columbia \\ University College of Physicians and \\ Surgeons, New York, USA
}

\begin{abstract}
Noninvasive ventilation has been utilized successfully in the pre- and out-of-hospital settings for a variety of disorders, including respiratory distress syndrome in neonates, neurologic and pulmonary diseases in infants and children, and heart failure as well as chronic obstructive pulmonary disease in adults. A variety of interfaces as well as mechanical positive pressure devices have been used: simple continuous positive airway pressure devices are available which do not require sophisticated equipment, while a broad spectrum of ventilators have been used to provide bilevel positive airway pressure. Extensive training of transport teams may be important, particularly when utilizing bilevel positive airway pressure in infants and children.
\end{abstract}

\section{Introduction}

Noninvasive ventilation (NIV), often delivered as bilevel positive airway pressure (PAP), has been used successfully in neonates, infants, children, and adults with acute and/or chronic respiratory failure. Though not a true mode of ventilation, continuous positive airway pressure (CPAP) has also proven quite useful in some patients with respiratory failure, and is often included in discussions of NIV. NIV may help avoid tracheal intubation, 1-4 $^{-4}$ and may also help preserve pharyngeal function including speech and swallowing abilities during an episode of respiratory failure; 4 it may also help avoid other complications related to invasive ventilation, $, 5,6$ and its use has been associated with a decrease in mortality in selected populations. ${ }^{3,7}$

In addition, CPAP for adults with acute respiratory failure in the prehospital setting has been associated with a decreased rate of intubation as well as mortality when compared to usual care. ${ }^{8}$ Both CPAP and bilevel PAP have been utilized during medical transport and in the prehospital setting for patients at various ages, and with various diseases. We reviewed the use of NIV- including both CPAP and bilevel PAP- during pre- and out-of-hospital care. The use and utility of NIV in this regard is increasing, in part as this therapy has been used extensively on patients with chronic disease who live at home. In addition, the utility of NIV as a primary therapy for many types of acute respiratory failure is expanding.

\section{Epidemiology}

Well-recognized benefits of NIV compared to invasive mechanical ventilation (simplicity of application, decreased need for sedation, and ability to rapidly discontinue as needed) suggest a potential role for NIV in pre- and out-ofhospital settings. Early experience with NIV in these settings suggests there are differences in its utility depending in part on patient age, and reflecting the different spectrum of diseases common to different age groups.

It is likely that early application of NIV contributes to success in patients with respiratory distress and incipient failure. The presence of dyspnea, tachypnea, and the use of accessory muscles of respiration are good indicators of respiratory distress at any age, and help to identify patients who may benefit from NIV. Contraindications to the use of NIV should be familiar to practitioners (Table 1).

\section{Pediatric vs adult patients}

Interhospital transport of neonates, infants, and children presents a similar situation compared to the prehospital setting. Many neonates, infants, or children require prompt referral to regional centers capable of providing more advanced therapies, and transport may be initiated in the clinic or the emergency or inpatient ward of a clinic or hospital. NIV may be initiated as a new therapy prior to such transport, or continued during transport as chronic therapy.

Reports of successful interhospital ground transport utilizing CPAP in neonates and infants include several series from Great Britain, $, 9,10$ as well as a larger series $(n=207)$ from Australia.11 This last report included mainly neonates and infants with respiratory distress syndrome, but also included some with bronchiolitis, chronic lung disease, and congenital heart disease. 11

A small series of infants and children $(\mathrm{n}=25)$, most with neurologic or pulmonary disease, were treated with CPAP or bilevel PAP during interhospital transport: patients had a broad spectrum of disorders commonly encountered in pediatric critical care, though no one had acute respiratory distress syndrome. 12 Around $1 / 3$ of these patients were NIV-dependent (mostly bilevel PAP) prior to transport: most of these had severe, chronic
Correspondence: Thyyar M. Ravindranath, Children's Hospital of New York-Presbyterian, 3959 Broadway, CHN 10-27, New York, NY 10032, USA.

E-mail: tr2148@columbia.edu

Key words: bilevel positive airway pressure, continuous positive airway pressure, noninvasive ventilation, transport, prehospital care.

Conflict of interests: all authors declare that there are no conflict of interests.

Received for publication: 4 August 2011.

Revision received: 4 January 2012.

Accepted for publication: 20 January 2012.

This work is licensed under a Creative Commons Attribution NonCommercial 3.0 License (CC BYNC 3.0).

(C) Copyright J.S. Baird and T.M. Ravindranath, 2012 Licensee PAGEPress, Italy

Pediatric Reports 2012; 4:e17

doi:10.4081/pr.2012.e17

neurologic disease, and constitute a growing population of inpatients with respiratory failure in some pediatric intensive care units; ${ }^{13}$ transport bilevel PAP capability may help smooth their transition from outpatient to inpatient (and back again). Though there were no significant complications, over $1 / 4$ of all transports required the use of advanced pediatric airway skills by the transport personnel (airway suctioning, bag-valve-mask ventilation, and cough-assist therapies).

Acute cardiogenic pulmonary edema in adults responds well to NIV in the pre- or outof-hospital settings:2,14-19 CPAP appears to be as or more effective than bilevel PAP in these patients, though both are associated with decreased work of breathing and increased cardiac output. ${ }^{20}$ More recently, it appears that adults with a variety of causes for acute respiratory failure, including chronic obstructive pulmonary disease and asthma, as well as those with cardiogenic pulmonary edema, benefit from CPAP compared to routine care: the need for tracheal intubation is decreased, as is mortality. 8 The application of NIV in the preand out-of-hospital settings is likely to be much broader than currently appreciated. ${ }^{21}$ Additional patient populations likely to benefit from out-of-hospital NIV include those requiring generous pain control and/or anxiolysis (in whom appropriate therapy in the pre- or out-ofhospital settings is likely to improve outcome), ${ }^{22}$ and patients with limitations in lifesustaining treatments including those requiring end-of-life care. 23

\section{Air transport and noninvasive ven- tilation}

CPAP has been used successfully in some 
neonates (most with neonatal respiratory distress syndrome) during air transport.11 Experience with CPAP for adults with obstructive sleep apnea during routine commercial airline operation is growing: guidelines governing in-flight use are common, ${ }^{24}$ and some commercial providers offer explicit guidance on their websites. ${ }^{25}$

Consideration regarding the use of NIV during air transports should focus on the limitations in space, equipment and personnel associated with the use of aircraft, as well as altitude physiology: gas in a closed space will expand in volume with altitude, as oxygen tension, as well as humidity and temperature, decline. The effect of an expanding collection of inspired gas in the stomach of a child newly introduced to NIV, or of decreasing humidity of inspired gas in the same patient with viscous, drying airway secretions- may be difficult to predict.

\section{Practice of noninvasive ventila- tion during pre- and out-of- hospital care}

The technology of NIV continues to evolve, and some terminology may be confusing; informative reviews of NIV are available which clarify most aspects of this therapy. ${ }^{4,26}$ The actual practice of NIV in the pre- or out-of-hospital setting is dependent on the availability of adequately trained transport personnel, monitoring capabilities, as well as specific equipment.

\section{Technology}

The provision of NIV requires a source of gas under pressure, tubes to transport that gas, as well as a patient interface device. Provision of NIV in the pre- and out-of-hospital settings thus may require large reserves of high-pressure oxygen and/or air, which could limit the use of NIV during transports of long duration. Desirable device characteristics for NIV transport devices include durability, lightweight and compact size, as well as compatibility with available types of power. Airway circuits that are easy to use and compatible with other respiratory care equipment likely to be encountered are best. Understanding and experience with the different modes, interface devices, and appropriate use are important to successfully apply NIV during transport in both acute and chronic respiratory failure.

CPAP is applied to spontaneously breathing patients: a constant airway pressure is delivered during inspiration and expiration. CPAP keeps alveoli open during exhalation and favors gas exchange during the entire respiratory cycle, thus helping to improve oxygenation
Table 1. Contraindications to noninvasive ventilation.

\begin{tabular}{|c|c|}
\hline Relative contraindications & Absolute contraindications \\
\hline Agitation and/or decreased level of consciousness & Cardiorespiratory arrest \\
\hline Barotrauma & Facial or neck trauma or significant recent surgery \\
\hline Hemodynamic instability & Massive hemoptysis or gastrointestinal hemorrhage \\
\hline High risk of aspiration & Upper airway obstruction (complete) \\
\hline \multicolumn{2}{|l|}{ Inability to protect airway } \\
\hline Inability to tolerate an interface device & \\
\hline
\end{tabular}

via an improvement in ventilation-perfusion matching; it may also contribute to a re-distribution of lung water. Cardiovascular effects may include a decrease in preload by reducing venous return as well as a decrease in afterload by a reduction in left ventricular systolic wall stress; patients with heart failure and hypervolemia often manifest a decrease in pulmonary congestion and an increase in cardiac output with CPAP. ${ }^{27}$ The optimal level of CPAP for patients at different ages is not precisely known, and depends in large part on the underlying disease and its severity.

Positive end expiratory pressure (PEEP) valves may be used to provide CPAP when appropriately attached to an interface device (usually a face mask), and are often able to accommodate variable gas flow. These simple devices do not regulate oxygen or air flows, or specify an oxygen concentration. Boussignac CPAP is a lightweight, disposable plastic device that contains no mechanical valve or sensor, and may be attached to a face mask or tracheostomy. A flow of oxygen and/or air accelerates via four parallel microchannels creating a flow dependent pressure in the plastic tube. Boussignac CPAP has been successfully used by personnel unfamiliar with conventional mechanical ventilators ${ }^{28}$ as well as in the emergency department for adults with cardiogenic pulmonary edema, ${ }^{29}$ and a recent trial suggests that it may have utility in the pre- or out-of-hospital setting. ${ }^{30}$ CPAP may also be provided in the absence of a ventilator using a bubble CPAP device: mechanical oscillatory vibrations are transmitted into the lungs as a result of non-uniform flow of gas bubbles from an underwater seal. In addition to devices able to provide CPAP only, numerous mechanical ventilators are able to provide CPAP while also offering the flexibility of using bilevel PAP when needed.

Bilevel PAP is used in patients requiring assistance throughout the respiratory cycle. For the most part, pressure-limited ventilators are used to provide NIV, resulting in bilevel PAP. Commonly used modes of bilevel PAP include both controlled and assisted ventilation: controlled bilevel PAP is time-triggered, while assisted bilevel PAP is triggered by the patient (by an increase in flow or a fall in airway pressure) and cycled as flow decreases or airway pressure increases beyond some limit,
Table 2. Noninvasive ventilation interface devices.

Face mask

Helmet

Mouthpiece

Nasal mask

Nasal pillows

Nasal prongs

or in a timed fashion. As pressure-limited ventilators deliver a preset pressure during inspiration and the delivered tidal volume depends on airway impedance, any leak in the system is compensated by an increase in the flow and any obstruction in the circuit or in the airway results in a decrease in the delivered volume. Pressure-limited ventilators vary widely in their ability to compensate for air leaks. ${ }^{31}$ The pressure support mode is generally better tolerated than pressure control, and provides a set pressure upon patient triggering which commonly allows the patient to control both inspiratory and expiratory times. BiPAP Andover, MA) provide bilevel PAP using spontaneous and timed modes of ventilation, analogous to pressure support and control mode settings on other devices.

\section{Equipment}

NIV interface devices include masks (nasal or face) as well as nasal prongs and pillows; rarely, mouthpieces have been utilized (Table 2 ). Full face masks may be poorly tolerated in some patients, and their use requires close observation to ensure that vomiting does not result in aspiration; frequently, however, they provide a better seal than nasal masks, thus avoiding major leaks and resultant NIV intolerance. Masks may be vented or nonvented; nonvented masks fixed tightly to the patient are utilized with a device which has an exhalation valve, permitting release of carbon dioxide without (open) exhalation ports. When used with vented masks, devices with an exhalation valve may autocycle. Full face mask improves efficacy by decreasing leaks and is appropriate for use in the setting of hypoxemic respiratory failure. Helmet interface devices serve as oxygen head tents and provide a predictable condevices (Respironics Inc/Philips Healthcare, 
Table 3. Devices used to provide continuos positive airway pressure and/or bilevel positive airway pressure in prehospital setting or during medical transport.

\begin{tabular}{|c|c|c|c|}
\hline Device & Interface device & Age range & Reference \\
\hline $\begin{array}{l}\text { Bio-Med Devices MVP-10 } \\
\text { (Guilford, CT) }\end{array}$ & Nasal prongs & $\mathrm{N}$ & 12 \\
\hline $\begin{array}{l}\text { Drager Babylog } 2000 \\
\text { (Telford, PA) }\end{array}$ & Nasal prongs & $\mathrm{N}, \mathrm{I}$ & 9 \\
\hline $\begin{array}{l}\text { Drager Oxylog } 3000 \\
\text { (Lubeck, Germany) }\end{array}$ & Face mask & A & 14 \\
\hline $\begin{array}{l}\text { Electro Medical Equipment Ltd } \\
\text { Infant Flow Driver } \\
\quad(\text { Brighton, Sussex, UK)* }\end{array}$ & Nasal prongs & $\mathrm{N}$ & 10 \\
\hline $\begin{array}{l}\text { Pulmonetics LTV-1000 } \\
\text { (Minneapolis, MN) }\end{array}$ & Nasal prongs, nasal mask & $\mathrm{I}, \mathrm{C}$ & 12 \\
\hline $\begin{array}{l}\text { Respironics Whisperflow } \\
\text { (Prospect Park, PA)* }\end{array}$ & Face mask & A & 2,8 \\
\hline $\begin{array}{l}\text { Respironics BiPAP S/T } \\
\text { (Prospect Park, PA) }\end{array}$ & Face mask & A & 19 \\
\hline $\begin{array}{l}\text { Stephan ventilator } \\
\text { (Saarbrucken, Germany) }\end{array}$ & Nasal prongs & $\mathrm{N}, \mathrm{I}$ & 11 \\
\hline
\end{tabular}

Table 4. Common complications of noninvasive ventilation.

\section{Common complications}

Aspiration pneumonia

Gastric distention

Hypercarbia

Interface device leaks and intolerance

Pressure sores or lesions

Ventilator asynchrony

centration of oxygen via CPAP. These devices may obviate some of the problems related to skin breakdown with prolonged NIV use via other interface devices; they have been used successfully on adults in the prehospital setting. 18

Many currently available ventilators are portable, lightweight, durable, and able to work either with a battery or commonly available electric power sources; these are important criteria to consider when selecting ventilators for use outside the hospital. Additional features generally include settings for a backup (or apnea) rate and a variety of sophisticated alarm capabilities. Some ventilators also offer the ability to set more complex parameters, including flow characteristics and leak compensation; they may also offer spirometry graphics. NIV has been provided during pre- or out-of-hospital care using a variety of devices (Table 3). No preference for a particular ventilator is apparent in the currently available literature, and the authors have participated in transports utilizing other NIV devices than those listed in Table 3.2,8,9,10-12,14,19

Respiratory frequency in a pressure control (or timed) mode is often set higher than the patient's own respiratory efforts in order to avoid unsupported, spontaneous breaths. The trigger sensitivity for pressure support breaths may need frequent adjustment, particularly in pediatric patients who may not generate as much flow or pressure drop with each breath compared to adults. For most pediatric patients, a pressure of $4-6 \mathrm{~cm}$ of water is sufficient as an initial CPAP setting, while a pressure of $10 \mathrm{~cm}$ of water has been used frequently in adults.2,8 Typical initial settings for bilevel PAP include an inspiratory pressure between 8-12 $\mathrm{cm}$ of water and expiratory pressure of 3$5 \mathrm{~cm}$ of water. The inspiratory pressure is gradually adjusted to a level that overcomes the work of breathing, while the expiratory pressure is set to achieve upper airway patency at end-expiration and restore adequate functional residual capacity, as reflected by improved oxygen saturation. The fractional concentration of oxygen is widely variable (from 0.21 up to 1) in patients treated with NIV during transport, 11,12 depending on the underlying pathology. Additional NIV equipment concerns include the use of humidification devices and compatibility with aerosol medication administration. Humidification is more important for infants and young children, and may be provided by heated or unheated passover devices, pass through devices, and heat and moisture exchangers. Many bilevel PAP devices incorporating continuous flow circuits allow the administration of nebulized medications without a change in delivered pressures.

\section{Transport personnel}

Transport personnel providing NIV are generally paramedics; a physician, nurse, and/or respiratory therapist may be included on pediatric transports. However, nurses- not para- medics- are responsible for neonatal transports in some locales. Transport personnel need specific educational programs that include comprehensive respiratory care training with practical examinations, annual training updates (which may include simulation training), as well as familiarity with local NIV transport protocols.8,12 Existing protocols 26 may be used as a springboard for the development of local NIV transport protocols.

Transport personnel who utilize NIV in acutely ill adults may believe that NIV in pre- or out-of-hospital settings is safe and easy to use, ${ }^{19}$ while those who utilize NIV in sick infants or children may suggest the need to be particularly well-trained and experienced. 12 The sensation of positive pressure and the anxiety and distress associated with underlying disease are often difficult for these patients to tolerate; patience and reassurance, as well as careful, continuous monitoring are essential to the success of NIV in children. Gradual increases in airway pressure, alternative interface devices, and care to appropriately recognize and treat complications (Table 4) will be helpful in order to increase NIV success. Additional training for pediatric providers may be important (though data are limited); bilevel PAP capability in these teams mandates the ability to address the problem of excessive airway secretions and the inability of young patients to clear them. Transport personnel should therefore be trained in the appropriate use of devices to clear airway secretions, including suction and bag-valvemask ventilation, as well as cough-assist devices. The ability to recognize progressive respiratory failure during NIV is essential, as it may establish the need for tracheal intubation with invasive mechanical ventilation.

\section{References}

1. Pang D, Keenan SP, Cook DJ, Sibbald WJ. The effect of positive pressure airway support on mortality and the need for intubation in cardiogenic pulmonary edema: a systematic review. Chest 1998;114:118592.

2. Hubble MW, Richards ME, Jarvis R, et al. Effectiveness of prehospital continuous positive airway pressure in the management of acute pulmonary edema. Prehosp Emerg Care 2006;10:430-9.

3. Peter JV, Moran JL, Phillips-Hughes J, et al. Effect of non-invasive positive pressure ventilation (NIPPV) on mortality in patients with acute cardiogenic pulmonary oedema: a meta-analysis. Lancet 2006; 367:1155-63.

4. Hillberg RE, Johnson DC. Noninvasive ventilation. N Engl J Med 1997;337:1746- 


\section{2.}

5. Bercault N, Boulain T. Mortality rate attributable to ventilator-associated nosocomial pneumonia in an adult intensive care unit: a prospective case-control study. Crit Care Med 2001;29:2303-9.

6. Devlin JW, Nava S, Fong JJ, et al. Survey of sedation practices during noninvasive positive-pressure ventilation to treat acute respiratory failure. Crit Care Med 2007;35: 2298-302.

7. Brochard L, Mancebo J, Wysocki M, et al. Noninvasive ventilation for acute exacerbations of chronic obstructive pulmonary disease. N Engl J Med 1995;333:817-22.

8. Thompson J, Petrie DA, Ackroyd-Stolarz S, Bardua DJ. Out-of-hospital continuous positive airway pressure ventilation versus usual care in acute respiratory failure: a randomized controlled trial. Ann Emerg Med 2008;52:232-41.

9. Bomont RK, Cheema IU. Use of nasal continuous positive airway pressure during neonatal transfers. Arch Dis Child Fetal Neonatal Ed 2006;91:F85-9.

10. Simpson JH, Ahmed I, McLaren J, Skeoch $\mathrm{CH}$. Use of nasal continuous positive airway pressure during neonatal transfer. Arch Dis Child Fetal Neonatal Ed 2004; 89:F374-5.

11. Murray PG, Stewart MJ. Use of nasal continuous positive airway pressure during retrieval of neonates with acute respiratory distress. Pediatrics 2008;121:e754-8.

12. Baird JS, Spiegelman JB, Prianti R, et al. Noninvasive ventilation during pediatric interhospital ground transport. Prehosp Emerg Care 2009;13:198-202.

13. Graham RJ, Dumas HM, O'Brien JE, Burns JP. Congenital neurodevelopmental diagnoses and an intensive care unit: defining a population. Pediatr Crit Care Med
2004;5:321-8.

14. Weitz G, Struck J, Zonak A, et al. Prehospital noninvasive pressure support ventilation for acute cardiogenic pulmonary edema. Eur J Emerg Med 2007;14:276-9.

15. Kallio T, Kuisma M, Alaspaa A, Rosenberg PH. The use of prehospital continuous positive airway pressure treatment in presumed acute severe pulmonary edema. Prehosp Emerg Care 2003;7:209-13.

16. Kosowsky JM, Stephanides SL, Branson RD, Sayre MR. Prehospital use of continuous positive airway pressure (CPAP) for presumed pulmonary edema: a preliminary case series. Prehosp Emerg Care 2001;5:190-6.

17. Gardtman M, Waagstein L, Karlsson T, Herlitz J. Has an intensified treatment in the ambulance of patients with acute severe left heart failure improved the outcome? Eur J Emerg Med 2000;7:15-24.

18. Foti G, Sangalli F, Berra L, et al. Is helmet CPAP first line pre-hospital treatment of presumed severe acute pulmonary edema? Intensive Care Med 2009;35:656-62.

19. Craven RA, Singletary N, Bosken L, et al. Use of bilevel positive airway pressure in out-of-hospital patients. Acad Emerg Med 2000;7:1065-8.

20. Mehta S, Al-Hashim AH, Keenan SP. Noninvasive ventilation in patients with acute cardiogenic pulmonary edema. Respir Care 2009;54:186-95.

21. Taylor DM, Bernard SA, Masci K, et al. Prehospital noninvasive ventilation: a viable treatment option in the urban setting. Prehosp Emerg Care 2008;12:42-5.

22. Hatlestad D. Pain management in the field. Emerg Med Serv 2001;30:64-70.

23. Duchateau FX, Beaune S, Ricard-Hibon A, et al. Prehospital noninvasive ventilation can help in management of patients with limitations of life-sustaining treatments. Eur J Emerg Med 2010;17:7-9.

24. Walker J, Kelly PT, Beckert L. Airline policies for passengers with obstructive sleep apnoea who require in-flight continuous positive airways pressure. Respirology. 2010;15:556-61.

25. Walters G, James A, Gill S, Banerjee D. Airline acceptability of CPAP: is relevant information available on airlines' websites? Prim Care Respir J 2010;19:87-8.

26. Mehta S, Hill NS. Noninvasive ventilation. Am J Respir Crit Care Med 2001;163:54077.

27. Pinsky MR, Summer WR, Wise RA, et al. Augmentation of cardiac function by elevation of intrathoracic pressure. J Appl Physiol 1983;54:950-5.

28. Dieperink W, Jaarsma T, van der Horst IC, et al. Boussignac continuous positive airway pressure for the management of acute cardiogenic pulmonary edema: prospective study with a retrospective control group. BMC Cardiovasc Disord 2007;7:40.

29. Moritz F, Brousse B, Gellee B, et al. Continuous positive airway pressure versus bilevel noninvasive ventilation in acute cardiogenic pulmonary edema: a randomized multicenter trial. Ann Emerg Med 2007;50:666-75.

30. Templier F, Dolveck F, Baer M, et al. Boussignac continuous positive airway pressure system: practical use in a prehospital medical care unit. Eur J Emerg Med 2003;10:87-93.

31. Mehta S, McCool FD, Hill NS. Leak compensation in positive pressure ventilators: a lung model study. Eur Respir J 2001;17: 259-67. 